\title{
Design of an Electromagnet for Electron Cyclotron Resonance Plasma System for Deposition of Fluorine Doped Tin Oxide
}

Received September 7, 2020; revised November 1, 2020; accepted November 3, 2020

\author{
Bup Ju Jeon* \\ Department of Energy \& Environmental Engineering, Shinhan University, Dongducheon 11340, Republic of Korea
}

*Corresponding author E-mail: bjjeon@shinhan.ac.kr

\begin{abstract}
Electromagnets suitable for large-scale electron cyclotron resonance (ECR) plasma systems consisting of linear microwaves of $2.45 \mathrm{GHz}$ with a uniform electromagnetic field of $875 \mathrm{G}$ were designed from simulation data. A thin film of fluorine-doped tin oxide was deposited at low temperature using the designed electromagnet to obtain the desired electromagnetic field. The simulation data and numerical analysis results for the electromagnetic field of the designed electromagnet and the formation of a large-scale ECR zone agreed well. A large-scale ECR zone with a magnetic field of $875 \mathrm{G}$ was formed at $16 \mathrm{~cm}$ from the z-axis. The composition of the fluorine-doped tin oxide film deposited under the ECR zone of $16 \mathrm{~cm}$ from the z-axis was dependent on the input current of the electromagnet, which affected the deposition region and the ECR zone. Within this experimental range, the distribution of electrical resistivity of the thin film was influenced by the electromagnetic field.
\end{abstract}

Keywords: Electromagnet, Magnetic field, Electron cyclotron resonance plasma, Fluorine-doped tin oxide, Electrical resistivity

\section{Introduction}

Recently, the demand for portable touch-screen panels (PTSPs) in portable electronic devices has drastically increased. Therefore, transparent conducting oxides (TCOs), which possess high electrical conductivity and high optical transmission in the visible wavelength region, are being increasingly used. Indium tin oxide (ITO) is a typical TCO that is widely used for applications requiring good electrical conductivity, such as organic light-emitting devices, photovoltaic devices, and portable electronic devices [1-9]. Multi-component oxides with various dopants such as binary and ternary oxides of indium zinc oxide (IZO), aluminum zinc oxide (AZO), and fluorine-doped tin oxide (FTO) have been proposed as TCO alternatives to ITO. Some of them are also being partially commercialized [10-13]. These TCOs are expected to possess low electrical resistance and high transmission in the visible wavelength region to fabricate TSPs of outstanding image quality with a good and fast response to the input signal. Moreover, the TCOs should also be durable to facilitate operation under external impact and prolonged frigid conditions, allowing them to be employed in the manufacture of smart electronic devices with transparent heating and variable color functionality of the window. FTO is a potential candidate for a TCO because its visible transmission and electrical conductivity are comparable to those of ITO.

ITO is typically used in transparent conductive films, but there is a limit to its use for flexible solar cells or dye-sensitized solar cells because of its low resistance to acid. However, FTO has excellent resistance to acids, low electrical resistance, and high optical transmittance, making it a substitute for ITO. Further, unlike the film deposited by the sputtering or evaporation method, the film deposited by ECR metal-organic chemical vapor deposition (MOCVD) has excellent adhesion between polyethylene terephthalate (PET) substrates and the deposited FTO layer due to anchoring. The anchoring effect was studied previously by examining the deposition of $\mathrm{Cu}$ film using ECR MOCVD [14]. In addition, the ECR MOCVD method can be used at temperatures below $80{ }^{\circ} \mathrm{C}$; thus, it can be applied to polymer substrates through a low-temperature process. FTO is typically used as the electrode material in dye-sensitized solar cells (DSSCs) and smart windows due to its corrosion resistance against acid [15]. FTO exhibits a wide range of band gap energy depending on the concentration and composition of the dopant material. It also exhibits a high optical transmittance of $92 \%$ in the visible range of $400-700 \mathrm{~nm}$, and a low electrical resistivity of $5.7 \times 10^{-2} \Omega \mathrm{cm}[12,16]$. FTO requires a specific lowtemperature process that is used for depositing a low-melting-point metal oxide on a PET film via MOCVD. For this, the ECR plasma system is the most suitable.

The deposition of a FTO film using a large-scale ECR plasma system was previously reported [17]. Large circular electromagnets were employed for use in the ECR plasma system; however, they cannot be used commercially owing to space limitations. To achieve a large-scale ECR plasma system with high energy density, it is important to induce linear microwaves in the chamber and ensure a uniform distribution of the electromagnetic field.

In this study, an ECR system was designed from simulation data with linear microwaves of frequency $2.45 \mathrm{GHz}$ and an electromagnetic field distribution of $875 \mathrm{G}$, and the characteristics of the deposited FTO film were studied. 


\section{Experimental details}

It is important to design an electromagnet that can provide a uniform electromagnetic field and microwave-induced intensity at the substrate surface to form a large-scale ECR plasma system. Therefore, we used a microwave system composed of a dual-type microwave generator and microwave guide. As shown in Fig. 1, the large-scale ECR plasma system consists of two parts, namely the dual microwave generator and the electromagnet. Microwaves of $2.45 \mathrm{GHz}$ were introduced into the plasma chamber through a copper antenna of low electrical resistance in a $4 \mathrm{~cm}$ diameter quartz tube. The central portion of each rectangular electromagnet was hollow to facilitate the formation of a uniform electromagnetic field. The plasma chamber and reactor for ionization and decomposition of the reactants were double-walled and contained a cooling jacket of dimensions $85 \mathrm{~cm} \times$ $17.5 \mathrm{~cm} \times 51 \mathrm{~cm}$. The plasma chamber was located near the central portion of the rectangular electromagnets. Ar and tetra methyl tin (TMT) were used as the flow gas and metal organic (MO) source, respectively. For FTO deposition, the base pressure was maintained at $2.0 \times 10^{-6}$ Torr using three turbo-molecular pumps; the temperature of the canister with TMT was maintained at $-5{ }^{\circ} \mathrm{C} . \mathrm{O}_{2}$ was used as the reaction gas with a supply rate of $50 \mathrm{sccm}$. The vapor pressure of TMT in the canister was kept constant at 65 Torr for deposition of a certain thickness. $\mathrm{CF}_{4}$ gas was supplied at a rate of $3 \mathrm{sccm}$ for doping of Fluorine and was premixed with reaction gas to be supplied to the reactor. During the FTO deposition, the working pressure was maintained at $1.0 \times 10^{-3}$ Torr. The uniform deposition of the FTO film is affected by the uniformity of the reaction gas supplied. In the ECR MOCVD process, the source injection affects the flow pattern of the fluid in the vacuum process, which can change the uniformity of the deposited film. The nozzle used in this experiment was tested and optimized using three previously known types of nozzles [18].

The plasma gas and reactive gas were introduced by a linear nozzle of width $62.5 \mathrm{~cm}$. The nozzle had holes of diameter $0.05 \mathrm{~cm}$, which were approximately $1 \mathrm{~cm}$ apart on the $0.64 \mathrm{~cm}$ tube. The nozzle was placed at a suitable distance from the main drum. PET film was used as the substrate. An FTO film was deposited on a roll of PET film of width $50 \mathrm{~cm}$ and length $250 \mathrm{~m}$. The line speed of the main drum was 0.1 and $0.3 \mathrm{~m} / \mathrm{min}$. The main drum coiled with the PET film was located for the deposition with uniform electromagnetic field and microwave-induced intensity at the substrate surface. The PET film was designed to pass through the center of the main drum. Figure 1(b) depicts a schematic of the side view of the ECR plasma system. Three turbo-molecular pumps were installed to facilitate a uniform distri-

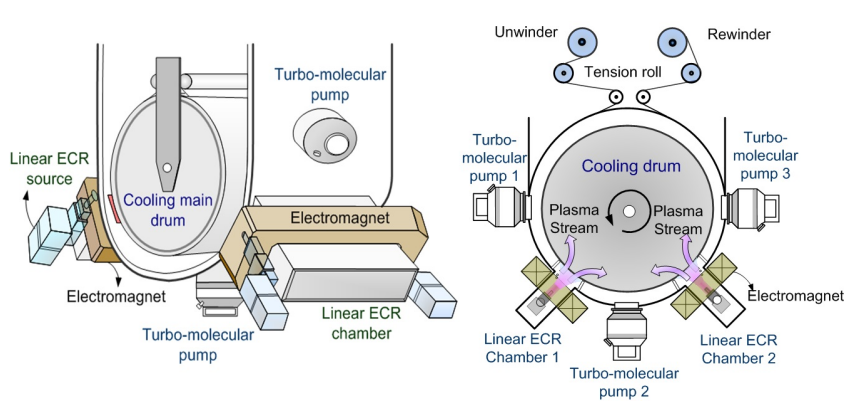

Figure 1. (a) 3D image and (b) side view of the large-scale ECR MOCVD plasma system with linear microwave and electromagnet. bution of the flow gas around the substrate and the exhaust gas between the ECR plasma generators.

A magnetic simulator consisting of a communications program for the transfer of data and a current controller with power supply was employed to design the large-scale electromagnet. The electromagnetic field simulations were measured by a gauss meter (Tesla Gauss meter TM701) equipped with an electromagnetic detector probe and jig for various coordinates. Electromagnetic fields were measured $0.5 \mathrm{~cm}$ apart up to $x=50 \mathrm{~cm}, y=12 \mathrm{~cm}$, and $\mathrm{z}=20 \mathrm{~cm}$ from the center of the electromagnet. The measured data was written in xyz contour using Origin software. The optical and electrical properties of the FTO thin film deposited on the PET film by the large-scale ECR plasma system were analyzed with varying electromagnetic currents and line speeds for deposition. To compare the optical and electrical properties, FTO thin films were deposited under the same pressure, microwave power, flow rate of carrier gas, and metal organic source gas conditions.

The composition and surface morphology of the deposited thin films were analyzed by electron spectroscopy for chemical analysis (ESCA, PHI 5800) and scanning electron microscopy (SEM, type Hitachi S-4200). A UV-vis spectrophotometer (Konica Minolta, CM-3500d) and four-point probe (Mitsubishi Analytec., Loresta-GP, MCP-T610, ESP-type probe) were employed to measure the optical transmittance and electrical surface resistance of the FTO thin film.

\section{Results and discussion}

The concentration profile of active ions around the given substrate is critical for uniform deposition on a large-scale substrate. The uniform deposition of the FTO thin film depends on the concentration profile of active ions generated from the ECR plasma and the flow pattern of the reactive gas supplied to the reactor chamber. While neutral radical species and molecules are affected by the pressure distribution in the deposition chamber, active ions and electrons are affected by the electromagnetic field. Low-temperature deposition using ECR plasma is affected by active ions, whose behavior is dependent on the distribution of the electromagnetic field; hence, a uniform magnetic field is crucial.

Figure 2 shows the position with the ECR zone of $875 \mathrm{G}$ from the electromagnet center, the electromagnetic planning, and the image installed. The specific position of the ECR zone with $875 \mathrm{G}$ depends on the current applied to the electromagnet. When the substrate is placed in the ECR zone, etching by the ion bombardment damages the surface of the substrate. When the substrate is adequately far away from the ECR zone, deposition occurs by the reactive ion species generated from the decomposition of the reaction gas by the ions and electrons generated in the ECR zone. Therefore, the ECR zone is a key factor in determining the etching and deposition conditions on the substrate surface. The distance of the substrate from the ECR zone of $875 \mathrm{G}$ along the electromagnetic width (x-axis) at an input magnet current of 292 A was in the range of $15-18 \mathrm{~cm}$. The ECR zone at the plasma chamber position of -20 to $20 \mathrm{~cm}$ (on the $\mathrm{x}$-axis) and -12 to 12 $\mathrm{cm}$ (on the y-axis) showed homogeneous broadening.

Figure 3 shows the magnetic field (Gauss) distribution contour obtained from the theoretical and experimental values at ECR plasma reactor dimensions of $85 \mathrm{~cm} \times 17.5 \mathrm{~cm} \times 51 \mathrm{~cm}$. It was simulated by varying the input current when the ECR plasma magnet dimension was from the center of the plasma chamber $(x=y=z=0)$ to $x=50$ $\mathrm{cm}, \mathrm{y}=12 \mathrm{~cm}$, and $\mathrm{z}=20 \mathrm{~cm}$ to obtain the uniform magnetic field. 


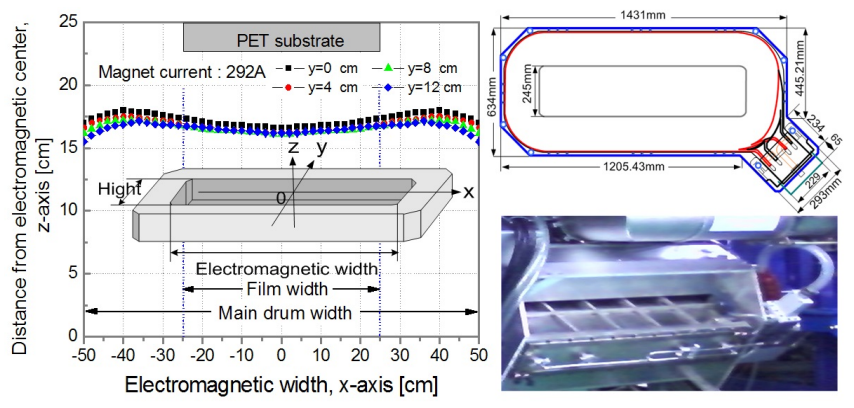

Figure 2. ECR zone of $875 \mathrm{G}$ along the electromagnet width (x-axis) at an input magnet current of $292 \mathrm{~A}$ and image of an electromagnet with dimensions of $143.1 \mathrm{~cm}$ $\times 63.4 \mathrm{~cm}$.
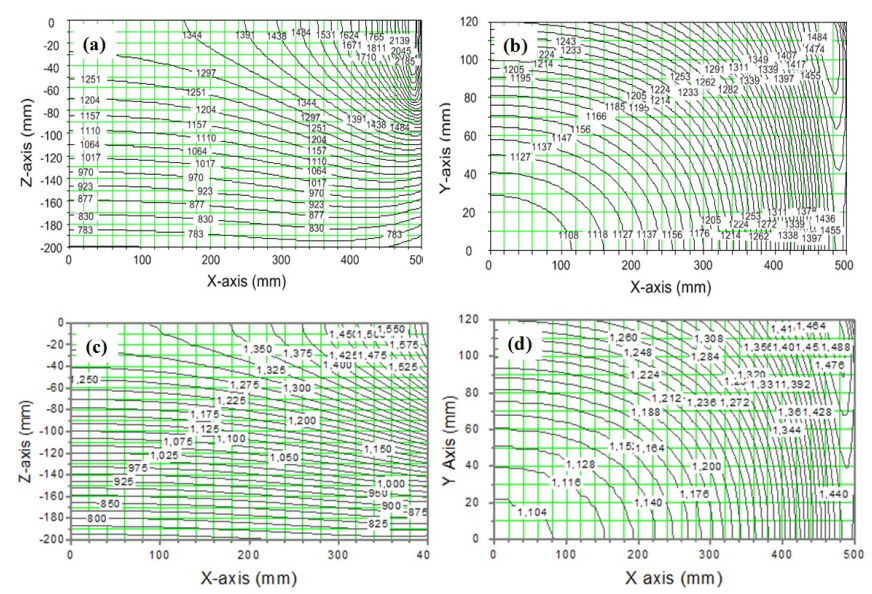

Figure 3. Comparison of (a), (b) simulated and (c), (d) measured total magnetic field (Gauss) distribution contour in the xz-plane, $y=0$.

The electromagnet is designed based on the simulation data. The magnetic field (Gauss) distribution contour shows a symmetrical size range of $x=50 \mathrm{~cm}, y=12 \mathrm{~cm}$, and $z=20 \mathrm{~cm}$ from the center of the plasma chamber $(x=y=z=0)$. Figures $3(a)$ and $3(b)$ show the simulated magnetic field (Gauss) distribution contour in the xz-plane and xy-plane, respectively. Figures 3(c) and 3(d) show the magnetic field (Gauss) distribution contour in the xz-plane and xy-plane from the designed electromagnet based on the simulation data in Figs. 3 (a) and 3(b), respectively. These results are in good agreement with the theoretical data. The ECR region of $875 \mathrm{G}$ is formed in the range of -16 to $-17 \mathrm{~cm}$ along the z-axis. The results illustrated in Fig. 3(a) are consistent with those in Fig. 2. The magnetic field is much higher at the center compared to lower side of the electromagnetic $\mathrm{z}$-axis. The magnetic field at the $500 \mathrm{~mm}$ of the $\mathrm{x}$-axis with a dense coil of electromagnet is more than $2000 \mathrm{G}$. The magnetic field contour in the xy-plane at the center of the electromagnetic $z$-axis has an elliptical orbit.

Figure 4 shows the simulated magnetic field distribution by moving the $y$-axis $10 \mathrm{~cm}$ away from the $\mathrm{z}$-axis from the center of the electromagnet. As shown in Fig. 4(a), the magnetic field increases around the edge of the electromagnet but decreases at the center of the electromagnet. The magnetic field of $875 \mathrm{G}$ meets the condition for ECR formation in the range of $0-25 \mathrm{~cm}$ on the $\mathrm{x}$-axis.

Figure 4 (b) shows the magnetic field distribution by changing the $\mathrm{z}$-coordinate at each position of the $\mathrm{x}$-axis. The magnetic field
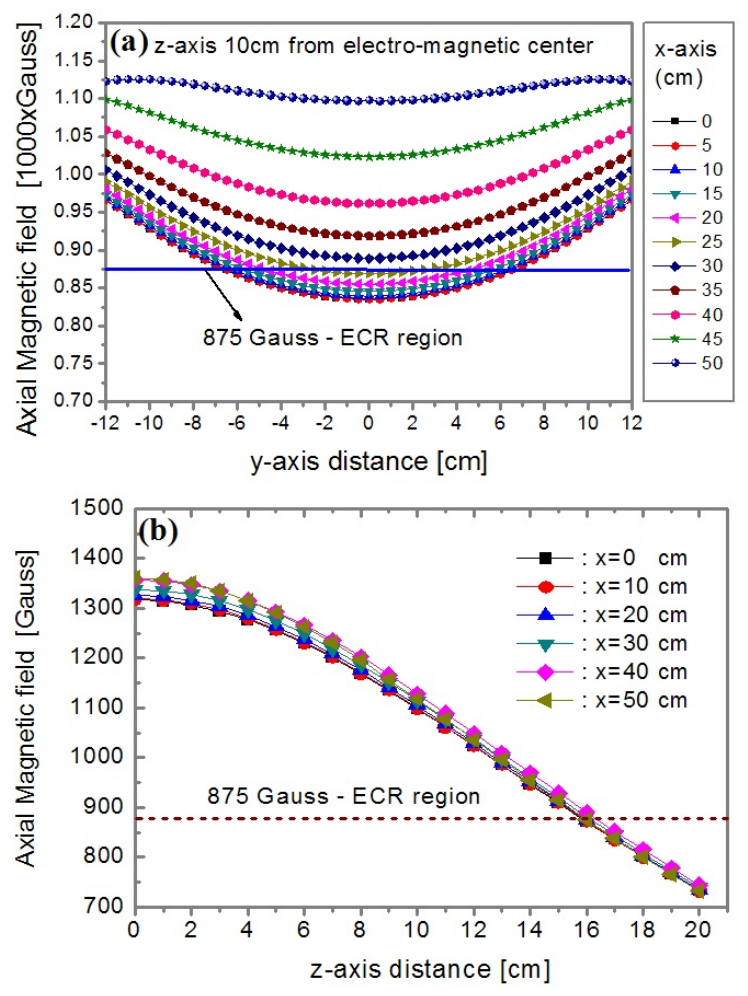

Figure 4. Magnetic field distribution with (a) $y$-axis (electromagnetic width) and (b) z-axis.

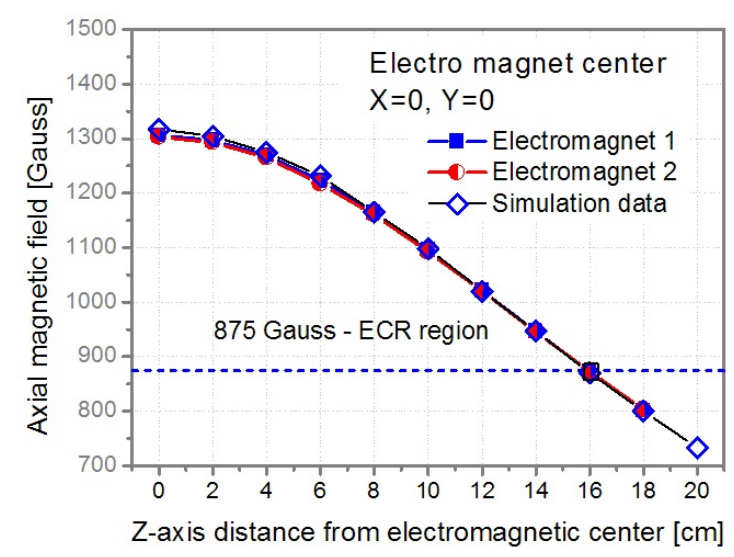

Figure 5. Comparison of magnetic field along the z-axis and ECR zone in the designed electromagnet 1,2 .

decreased dramatically as we moved further from the center of the $\mathrm{z}$-axis. The ECR region with a magnetic field of $875 \mathrm{G}$ on the $\mathrm{z}$-axis is the point corresponding to $16 \mathrm{~cm}$ from the center. Therefore, the points beyond $16 \mathrm{~cm}$ would be appropriate for the deposition process when using this electromagnetic.

As shown in Figs. 3 and 4, based on the simulation data of the electromagnetic field, an electromagnet with a magnetic field of $875 \mathrm{G}$ that satisfies the ECR condition was designed for the experiment. The electromagnet is formed of 14 layers with a small rectangular bandage copper tube, and cooling water flowing through the copper tube. The maximum input current and electrical potential are $300 \mathrm{~A}$ and $50 \mathrm{~V}$, respectively. A pressure drop of $800 \mathrm{kPa}$ and a flow rate of 0.0083 

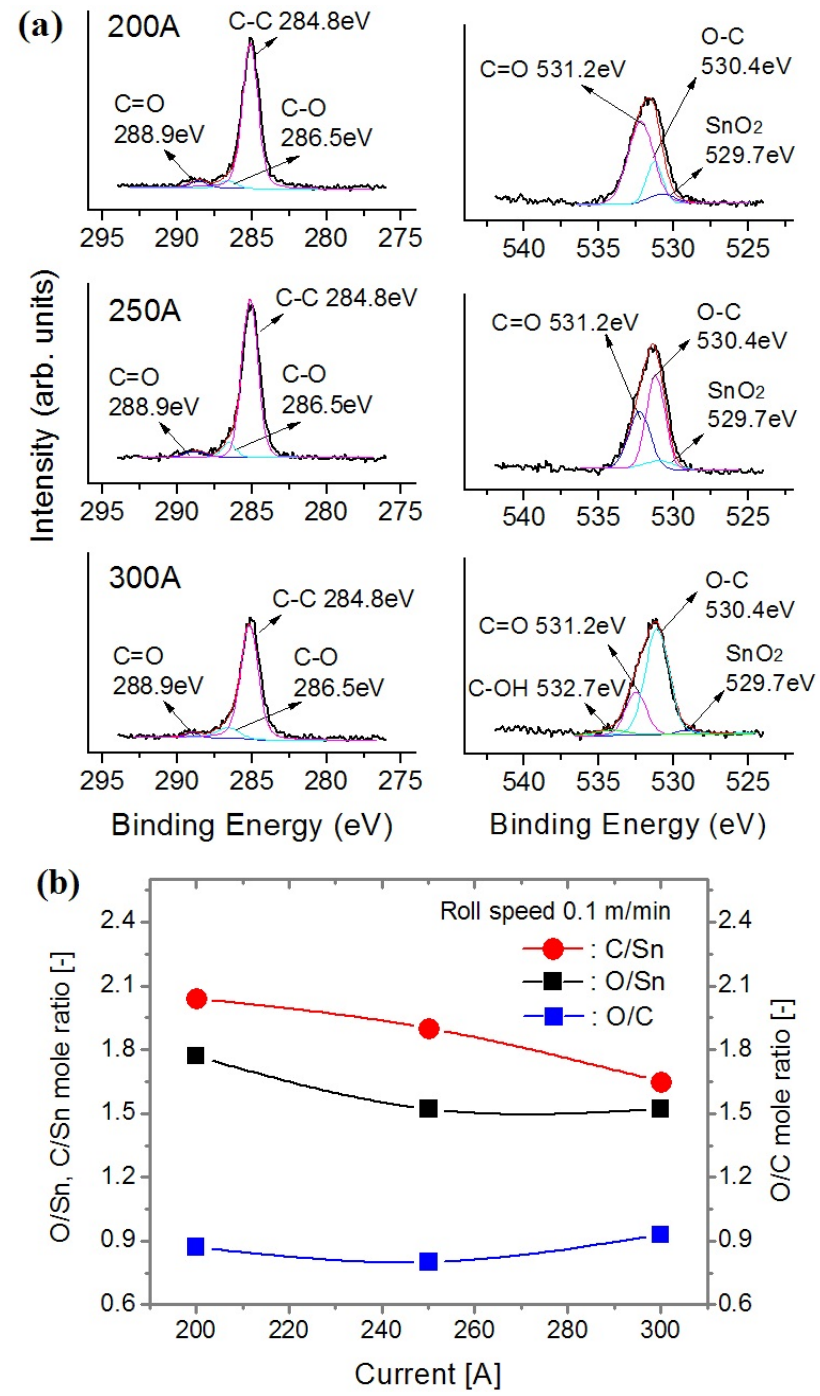

Figure 6. (a) XPS spectra and (b) mole ratio of deposited FTO film with input electromagnetic current.

$\mathrm{m}^{3} / \mathrm{min}$ were set in the rectangular copper tube. Figure 5 shows the simulated data and the actual measured electromagnetic field along the z-axis. The measured electromagnetic field is well consistent with the simulation data, and the ECR zone having a magnetic field of 875 $\mathrm{G}$ was formed at $16 \mathrm{~cm}$ along the $\mathrm{z}$-axis, as shown in Fig. 4(b).

FTO was deposited on the PET film by the ECR system configured by the dual microwave generator and designed a electromagnet. XPS analysis was performed to investigate the chemical composition of the deposited film. The properties of the deposited films, such as composition, changed because the input current of the electromagnet affected the deposition region and ECR zone. Figure 6 shows the narrow scan XPS spectra and the molar ratio of the deposited FTO films at a roll speed $0.1 \mathrm{~m} / \mathrm{min}$ and input currents of 200,250 , and 300 A. Figure 6(a) shows the intensity of the intermolecular bonds from the peak separation of C1s at a binding energy of $280-290 \mathrm{eV}$, and O1s in the range 525 to $535 \mathrm{eV}$ for the deposited FTO thin film at different values of currents. The C1s peak shows the three peaks due to the $\mathrm{C}-\mathrm{C}$ bond at $284.8 \mathrm{eV}, \mathrm{C}-\mathrm{O}$ bond at $286.51 \mathrm{eV}$, and $\mathrm{C}=\mathrm{O}$ bond at $288.9 \mathrm{eV}$. The $\mathrm{O} 1 \mathrm{~s}$ peak also shows four peaks due

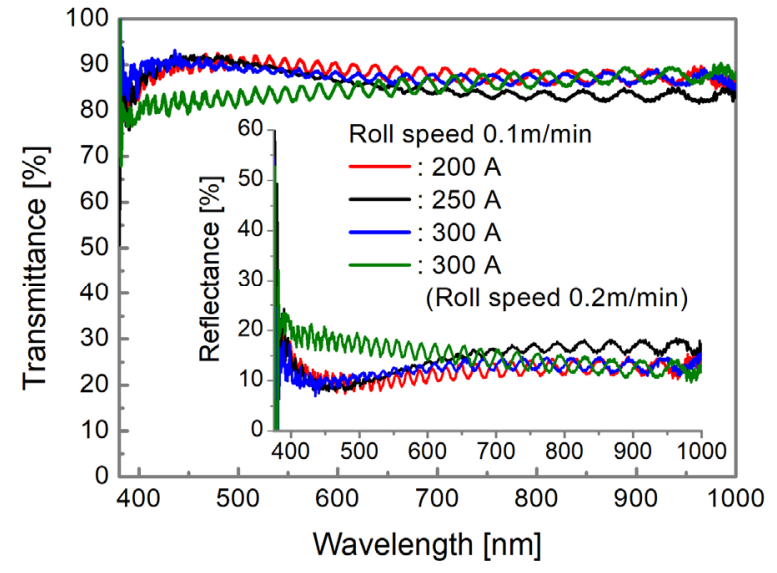

Figure 7. Optical transmittance and reflectance of the deposited FTO film with the electromagnetic input current.

to the O-Sn-O bond at $529.7 \mathrm{eV}, \mathrm{O}-\mathrm{C}$ bond at $530.4 \mathrm{eV}, \mathrm{C}=\mathrm{O}$ bond at $531.2 \mathrm{eV}$, and $\mathrm{C}-\mathrm{OH}$ bond at $532.7 \mathrm{eV}$. In the case of the carbon peak in the range $280-290 \mathrm{eV}$, the increase in electromagnetic current leads to an increase in the area of the $\mathrm{C}-\mathrm{O}$ peak at $286.51 \mathrm{eV}$ and a decrease in the area of the $\mathrm{C}=\mathrm{O}$ peak at $288.9 \mathrm{eV}$. The areas under the $\mathrm{O}-\mathrm{C}$ peak at $530.4 \mathrm{eV}$ and $\mathrm{C}-\mathrm{OH}$ peak at $531.2 \mathrm{eV}$ increased and the area under the $\mathrm{C}=\mathrm{O}$ peak at $531.2 \mathrm{eV}$ decreased. The position of the PET film substrate and ECR plasma zone depends on the electromagnetic current. Therefore, the electromagnetic current can affect the electrical and optical properties due to compositional changes in the deposited FTO film. To reveal the impact of the electromagnetic current on the compositional changes in the deposited FTO film, the atomic mole ratio was calculated from the peak separation in Fig. 6(a). Figure 6(b) shows the mole ratio of $\mathrm{O} / \mathrm{Sn}, \mathrm{C} / \mathrm{Sn}$, and $\mathrm{O} / \mathrm{C}$. The mole ratio of $\mathrm{O} / \mathrm{Sn}$ and $\mathrm{C} / \mathrm{Sn}$ decreases as the electromagnetic current increased. That is, the mole of Sn atoms in the deposited FTO films increased, and the mole ratios of $\mathrm{C}$ and $\mathrm{O}$ decreased. The mole ratios of $\mathrm{O} / \mathrm{C}$ did not change much with the electromagnetic current, but the mole of $\mathrm{O}$ atom increased at the current value of $300 \mathrm{~A}$. This is because the surface reaction to form the FTO film is efficiently conducted by the transfer of oxygen ions from the gas phase to the substrate as the current through the electromagnet increases.

Figure 7 shows a comparison between raw PET and the optical transmittance of the FTO film at conditions where the current of the electromagnet is 200, 250, and $300 \mathrm{~A}$. The raw PET used an optical film with an optical transmittance of $90.10 \%$ at a wavelength of 550 $\mathrm{nm}$. The transmittance of films deposited at 200, 250, and 300 A was $88.56,87.42$, and $84.32 \%$, respectively, and reflectivity was within the range of $10-20 \%$. The optical transmittance tends to decrease slightly with increasing the current in the electromagnet, which is believed to be influenced by the formation of dense film and by electrons with high energy as the ECR zone near the substrate.

Figure 8 shows the thickness of the FTO film deposited under 250 and 300 A conditions and the SEM image of the surface. An ionized ion is formed by the resonance of an electron cyclotron of an $\mathrm{O}_{2}$ gas supplied to the ECR area at a distance of more than $15 \mathrm{~cm}$ from the substrate under conditions of 250-300 A. Oxygen ions activated by the gas phase decomposition with reaction gas TMT from the nozzle and Sn ions formed FTO film. The thickness of the film decreased at the same deposition time as the current flowing through the 

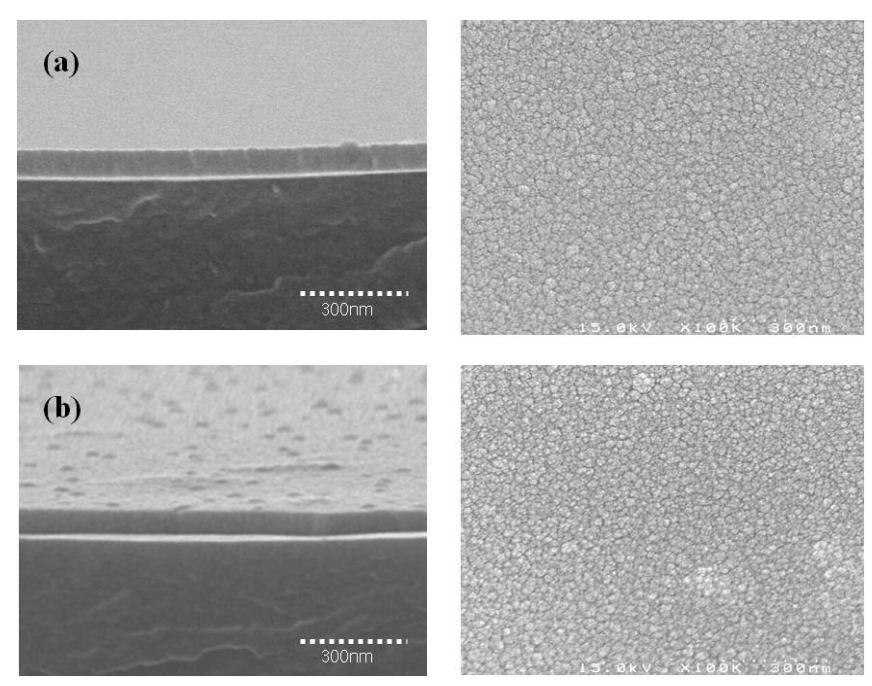

Figure 8. SEM images of cross-section and surface morphology at the electromagnetic input current (a) $250 \mathrm{~A}$ and (b) $300 \mathrm{~A}$.

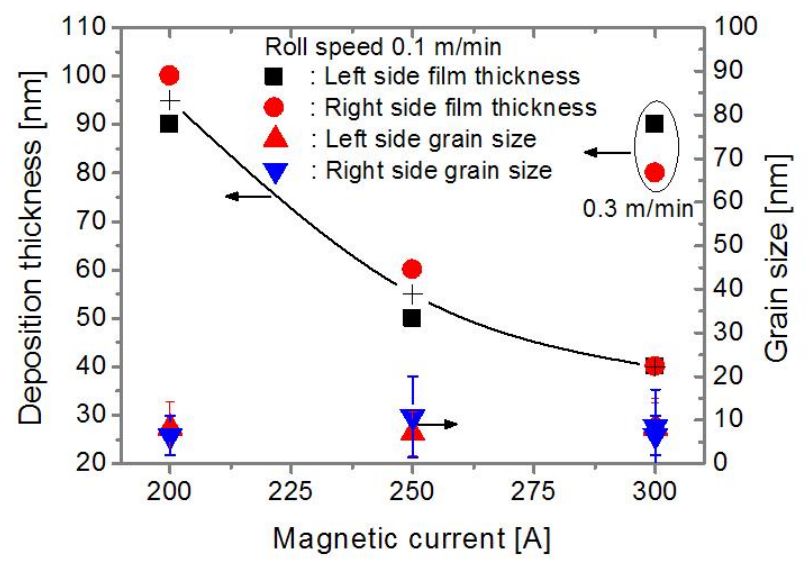

Figure 9. Deposition thickness and grain size from surface morphology of the deposited FTO film with the electromagnetic input current.

electromagnet increased due to the formation of the dense film. Surface morphology looked similar from the shape of grains present on the surface for synthesis condition of currents of 250 and $300 \mathrm{~A}$, but grain appeared to be smaller for that deposited at $300 \mathrm{~A}$. This is thought to be caused by the slight movement of the ECR zone towards the substrates by high current and the high energy of the active species by plasma density.

Figure 9 shows the film thickness and grain size on either edge of the deposited FTO film with the electromagnetic input current at line speed 0.1 and $0.3 \mathrm{~m} / \mathrm{min}$. In the case of the films deposited at the electromagnetic current of $200 \mathrm{~A}$, the film thickness on either edge was in the range of 90-100 $\mathrm{nm}$ and the thickness difference was approximately $10 \mathrm{~nm}$. For deposition conditions with current values of 250 and $300 \mathrm{~A}$, the thicknesses were 50-60 and $40 \mathrm{~nm}$, respectively. The film thickness on either edge of the deposited FTO film deposited at the current of $300 \mathrm{~A}$ was uniform. The grain sizes on the two edges of the deposited FTO films were 8.5 and $6.5 \mathrm{~nm}$ at $200 \mathrm{~A}, 7$ and 10.5 $\mathrm{nm}$ at $250 \mathrm{~A}$, and $8.5 \mathrm{~nm}$ at $300 \mathrm{~A}$. The grain size of the deposited FTO film at a high electromagnetic current had more uniform distribution. A possible explanation could be that the FTO films deposited at a low

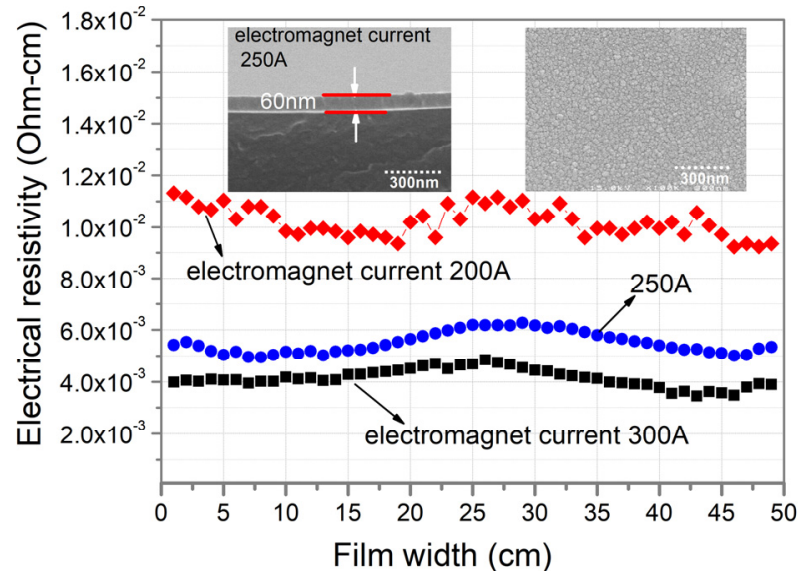

Figure 10. Surface resistance distribution with film width (x-axis) as a function of the input power magnetic field and SEM images of the cross-section and surface.

electromagnetic current were affected by the flow pattern of the active radicals due to the high deviance of the electromagnetic field. The FTO film deposited at a high electromagnetic current, however, was affected by the electromagnetic field rather than the flow pattern. That is, the reactive ions produced by the electromagnetic field at a high current were important for uniform film deposition on either edge of the deposited FTO film.

Figure 10 shows the surface electrical resistivity distribution and SEM images of the cross-section and the surface morphology of the deposited films. The thickness of the film deposited at the current value of $250 \mathrm{~A}$ was $60 \mathrm{~nm}$, and the surface morphology shows uniformly distributed fine grains with nano-scale sizes. The electrical resistivity was high. The electrical resistivity distribution of the film deposited at $200 \mathrm{~A}$ had high deviance in the range of $1.2 \times 10^{-2}$ to $9.0 \times$ $10^{-3} \Omega$-cm depending on the deposition position. At $250 \mathrm{~A}$, the surface electrical resistivity was much lower in the range of $5.5 \times 10^{-3}$ to $4.90 \times$ $10^{-3} \Omega$-cm on both edges of the deposited FTO film. The electrical resistivity distribution was higher at the center and lower parts at both the edges. This electrical resistivity distribution tendency is similar to the specific locations of the ECR zone with $875 \mathrm{G}$ in Fig. 2. This is attributed to the electrical resistivity distribution affecting the concentration of generated active ions from the decomposition of the reactive gas in the ECR zone. In the case of $300 \mathrm{~A}$ condition, the electrical resistivity showed a uniform distribution in the range of 4.8 $\times 10^{-3}-3.8 \times 10^{-3} \Omega$-cm influenced by the electromagnetic field more than that at $250 \mathrm{~A}$. As a result of this, the surface electrical resistivity improved and the electromagnetic field distribution became uniform in the ECR zone.

\section{Conclusions}

We designed an electromagnet for large-scale FTO film deposition using ECR plasma with a linear microwave of $2.45 \mathrm{GHz}$. The electromagnet having a uniform electromagnetic field distribution was simulated and it was specially produced based on the simulation data. The properties of the FTO film deposited at different applied currents were compared. The electromagnetic field at the designed electromagnetic zone was found to be consistent with the numerical analysis results, and an ECR zone having a magnetic field of $875 \mathrm{G}$ was formed at $16 \mathrm{~cm}$ from the $\mathrm{z}$-axis. The concentration of $\mathrm{O}, \mathrm{Sn}, \mathrm{F}$, and $\mathrm{C}$ in the 
fluorine-doped tin oxide film depended on the input electromagnetic current affecting the deposition region. The electrical resistivity of the deposited FTO film was dependent on the ECR zone.

\section{Acknowledgements}

This research was supported by the Small and Medium Business Administration (SMBA).

\section{References}

[1] K. Zhang, F. Zhu, C. H. A. Huan and A. T. S. Wee, J. Appl. Phys. 86, 974 (1999).

[2] M. D. Stoev, J. Touskova, and J. Tousek, Thin Solid Films 299, 67 (1997).

[3] F. Li, H. Tang, and J. Shinar, Appl. Phys. Lett. 70, 2741 (1997).

[4] J. C. Scott, J. H. Kaufman, P. J. Brock, R. DiPietro, J. Salem, and J. A. Goitia, J. Appl. Phys. 79, 2745 (1996).

[5] F. L. Wong, M. K. Fung, S. W. Tong, C. S. Lee, and S. T. Lee, Thin Solid Films 466, 225 (2004).

[6] Y. H. Liau and N. F. Scherer, J. Phys. Chem. 105, 3282 (2001).
[7] W. Wohlmuth and I. Adesida, Thin Solid Films 479, 223 (2005)

[8] C. J. Lee, H. K. Lin, C. H. Li, L. X. Chen, C. W. Wu, and J. C. Huang, Thin Solid Films 522, 330 (2012)

[9] A. Koppel, W. Kriegseis, B. K. Meyer, A. Scharmann, C. Daube, J. Stollenwerk, and J. Trube, Thin Solid Films 365, 139 (2000).

[10] H. L. Ma, X. T. Hao, J. Ma, Y. G. Yang, J. Huang, D. H. Zhang, and X. G. Xu, Appl. Surf. Sci. 191, 313 (2002).

[11] J. Loffler, R. Groenen, J. L. Linden, M. C. M. van de Sanden, and R. E. I. Schropp, Thin Solid Films 392, 315 (2001).

[12] T. Fukano and T. Motohiro, Sol. Energy Mater. Sol. Cells 82, 567 (2004).

[13] J. A. Jeong, H. K. Kim, H. W. Koo, and T. W. Kim, Appl. Phys. Lett. 103, 011902 (2013).

[14] B. J. Jeon, H. D. Ko, D. G. Byun, and J. K. Lee, Thin Solid Films 496, 395 (2006).

[15] C. Hudaya, J. H. Park, and J. K. Lee, Nanoscale Res. Lett. 7, 1 (2012).

[16] B. Thangaraju, Thin Solid Films 402, 71 (2002).

[17] B. J. Jeon, C. Hudaya, and J. K. Lee, J. Voc. Sci. Technol. A 34, 03151 (2016).

[18] B. J. Jeon, J. Nano. and Nanotech. 19, 1382 (2019). 